\title{
Safety and feasibility of laparoscopic versus open liver resection with associated lymphadenectomy for intrahepatic cholangiocarcinoma
}

\author{
Jia Wu ${ }^{1,}$, Junjun Han ${ }^{2,}$, Yuhua Zhang ${ }^{1}$, Lei Liang ${ }^{1}$, Junjun Zhao ${ }^{2}$, Fang Han ${ }^{1}$, Changwei Dou ${ }^{1}$, \\ Yuanbiao Zhang ${ }^{1}$, Jie Liu ${ }^{1}$, Weiding $\mathrm{Wu}^{1}$, Zhiming $\mathrm{Hu}^{1}$, Chengwu Zhang,* \\ ${ }^{1}$ Department of Hepatobiliary and Pancreatic Surgery \& Minimally Invasive Surgery, Zhejiang Provincial People's Hospital \& Hangzhou Medical \\ College affiliated People's Hospital, Hangzhou, Zhejiang, China; \\ ${ }^{2}$ Graduate Department, Bengbu Medical College, Bengbu, Anhui, China.
}

SUMMARY The safety and feasibility of laparoscopic versus open liver resection (LLR vs. OLR) associated lymphadenectomy for intrahepatic cholangiocarcinoma (ICC) are still controversial. The aim of the present study was to compare short and long-term outcomes. We reviewed data on 43 consecutive patients who underwent curative liver resection with associated lymphadenectomy for ICC. The short-term outcomes including postoperative morbidity and mortality, and the long-term outcomes including overall survival (OS) and recurrence-free survival (RFS) were compared. The median survival, 1- and 3-year OS in LLR and OLR groups were 22.5 months, $76.9 \%$ and $47.1 \%$, and 12.1 months, $43.1 \%$ and $20.0 \%$, respectively. The median survival, 1- and 3-year RFS in LLR and OLR groups were 10.3 months, $27.8 \%$ and $0 \%$, and 8.1 months, $24.0 \%$ and $4.0 \%$, respectively. The results showed that LLR obviously reduced intraoperative blood loss (median, $375 v s .500 \mathrm{ml}, p=0.016$ ) and postoperative hospital stay (median, $6 v s$. 9 days, $p=0.016$ ). Moreover, there was no significant difference in short-term outcomes including postoperative morbidity (including wound infection, bile leakage, liver failure and pneumonia) and mortality within 30 days, and long-term outcomes including OS and RFS between LLR and OLR. (all $p>0.05$ ). Multivariate analysis showed that CA19-9 level, TNM stage, and tumor differentiation were independent risk factors for OS and RFS. LLR for ICC is safety and feasibility compared with OLR. The advantage of LLR was to reduce intraoperative blood loss and postoperative hospital stay.

Keywords laparoscopy, intrahepatic cholangiocarcinoma, lymphadenectomy, liver resection.

\section{Introduction}

Intrahepatic cholangiocarcinoma (ICC) is the second most common primary liver cancer after hepatocellular carcinoma, which accounts for $10 \%$ to $20 \%$ of newly diagnosed liver cancers (1). The incidence of ICC has been rising on a global scale over the last twenty years, which may reflect both a true increase and the trend of earlier detection of the disease. Previous studies reported a 5 -year survival for ICC ranging from $15 \%$ to $40 \%$ (2). Several clinicopathologic parameters, including lymph node metastasis, vascular invasion and multiple tumors, have been raised as potential prognostic factors determining clinical outcomes $(3,4)$.

Liver resection remains the first-line curative treatment. Laparoscopic liver resection (LLR), which has progressed over the last 20 years, has become a feasible choice for various kinds of liver lesions owing to the development of high-tech surgical techniques and equipment. In 1995 and 1996, the minimally invasive liver resection series were reported $(5,6)$. Since then, a minimally invasive approach to liver resection has been used in the treatment of a myriad of conditions, and exponential dissemination has been experienced (7). However, the safety and feasibility of LLR for ICC are still controversial. Although ICC is not a contraindication for LLR, debates focusing on the risks of positive surgical margins, massive hemorrhage and difficulty with lymphadenectomy in LLR still exist. Nowadays, few reports referring to LLR for ICC are available (810). In 2015, Billy et al reported 11 patients with ICC underwent LLR, and 26 patients underwent open liver resection (OLR) (8). The results indicated that LLR was technically safe and the survival was comparable to OLR. However, most of them did not include enough relevant data, such as lymph node dissection, postoperative adjuvant chemotherapy, et al.

In the present study, we aimed to compare short- 
term outcomes including postoperative morbidity and mortality, and long-term outcomes including overall survival (OS) and recurrence-free survival (RFS) between LLR and OLR with associated lymphadenectomy for ICC.

\section{Materials and Methods}

\subsection{Patient selection}

A retrospective study was conducted on consecutive patients with ICC, who underwent curative liver resection and associated lymphadenectomy from Jan 2010 to Dec 2017 in Zhejiang Provincial People's Hospital, China. Curative liver resection was defined as removal of all microscopic and macroscopic tumors with a microscopically clear margin of surgical specimens (R0 resection). The resected tumors with surrounding liver tissues were examined histopathologically. Inclusion criteria were ( $i$ ) age between 18 and 80 years, (ii) ICC confirmed by postoperative pathological result; (iii) patients with associated lymphadenectomy; (iv) patients who received postoperative adjuvant chemotherapy. Exclusion criteria included: ( $i$ ) distal metastasis or macroscopic tumor thrombus in major portal/hepatic veins before operation, (ii) hilar cholangiocarcinoma or gallbladder cancer, (iii) a history of preoperative anticancer treatment, including biliary drainage. Informed consent was obtained from all the enrolled patients for their data to be utilized in clinical research. The present study was conducted in accordance with the Declaration of Helsinki and the Ethical Guidelines for Clinical Studies by the Institutional Ethics Committee of Zhejiang Provincial People's Hospital.

\subsection{Diagnosis and surgical procedure}

Enhanced computerized tomography (CT) or magnetic resonance (MR) were used to identify the type of tumor, relationship with adjacent tissue or organ and evaluate lymph node status routinely. Elevated carbohydrate antigen 19-9 (CA19-9) and carcinoma embryonic antigen (CEA) were another indicator for diagnosis of ICC. All patients underwent liver resection, with the intention of complete removal of macroscopic tumors, provided that the volume of the future liver remnant was estimated to be sufficient on CT or MR imaging volumetry. All patients were allocated into LLR or OLR group according to the different surgical approach. Major hepatectomy was defined as resection of three or more Couinaud's segments, while minor hepatectomy was resection of fewer than three segments.

For LLR, pringle maneuver was a commonly used method to block inflow of blood stream in the process of liver transection when severe bleeding occurred, which was implemented using an $8 \mathrm{~F}$ rubber catheter wrapping around hepatoduodenal ligament and tightening the catheter when necessary. Harmonic scalpel and Cavitron Ultrasonic Surgical Aspirator (CUSA) were employed during liver parenchymal transection. The branches of Glisson system or hepatic vein toward the resected liver were ligated by non-absorbable clips. Regional lymphadenectomy was carried out routinely, which included hepatoduodenal ligament lymph nodes (Site 12). Fine rubber tapes were employed to hang bile duct, hepatic artery and portal vein, which could make lymph node dissection easy. Resected specimens were put into a plastic bag and retrieved from the enlarged subumbilical incision. Typical liver resection and lymph node dissection is demonstrated in Figure 1.

All patients received six courses of postoperative preventive chemotherapy, which consisted of Gemcitabine (Day 1, Day 8) plus S-1 (Day1-14), cycled 3 weeks 6 times.

\subsection{Data collection and postoperative follow-up}

The patient- and liver-related variables included age, sex, comorbid illnesses (consists of diabetes mellitus, cardiovascular disease, chronic obstructive pulmonary disease, and renal dysfunction history), ASA score, abdominal surgical history, and preoperative serum total bilirubin (TBIL). The tumor-related variables included preoperative CA19-9 level, CEA level, maximum size of tumor, TNM stage by AJCC ( $8^{\text {th }}$ edition), nerve invasion, and tumor differentiation (well, moderately or poorly). The operative variables included range of hepatectomy (minor or major), number of lymphadenectomy ( $\geq 6$ or $<6$ ), intraoperative blood loss, and length of surgery.

The postoperative follow-up protocol included physical examination, serum tumor marker levels (CEA, CA19-9), CT or MR scan every month for the first 3 months, and then every 3 months for the initial 2 years and every 6 months for the following years. Recurrence and Metastasis were judged by PET/CT. The short outcomes including postoperative hospital stay, morbidity (including wound infection, bile leakage, liver failure and pneumonia) and mortality within 30 days. The long-term outcomes include OS and RFS. OS was calculated as the interval between the date of operation and death for any reason, with censoring at the date of last follow-up. RFS was calculated from the date of liver resection to the date of first ICC recurrence or the date of the last follow-up.

\subsection{Statistical analysis}

Statistical analysis was performed using SPSS statistical software (IBM SPSS, Inc., Chicago, IL, version 26.0). Survival curves were draw and compared by GraphPad (GraphPad Software, Inc. version 6.0). Continuous variables were presented as median \pm interquartile range (IQR), and compared between groups by Mann-Whitney $U$ test. Categorical variables were demonstrated as 
absolute numbers and compared between groups using the $\chi^{2}$ test. Kaplan-Meier method and log-rank test were employed to analyze the difference of OS and RFS between LLR and OLR groups. Statistical significance was inferred at a two-tailed $P$ value of $<0.05$.

\section{Results}

\subsection{Baseline characteristics}

A total of 43 patients undergoing curative liver resection and lymphadenectomy for diagnosed ICC met the inclusion criteria and were included. Among them, 18 patients received LLR, and 25 patients underwent OLR. The baseline characteristics of the patients in the two groups are listed in Table 1. Intraoperative blood loss of LLR group was less than OLR group (375 vs. $500 \mathrm{~mL}$, $p=0.016)$. There was no other difference between LLR and OLR in the aspect of the patient- and liver-related

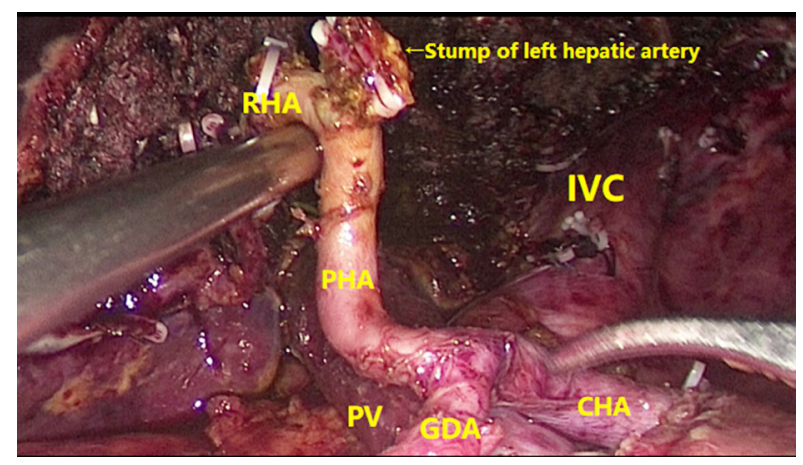

Figure 1. Typical figure of laparoscopic left hemi-hepatectomy and lymphadenectomy. RHA, right hepatic artery; IVC, inferior vein cava; PHA, primary hepatic artery; PV, portal vein; GDA, gastroduodenal artery; CHA, common hepatic artery. variables, the tumor-related variables and the operative variables.

\subsection{Comparisons of short-term outcomes}

The short outcomes including postoperative hospital stay, mortality (including wound infection, bile leakage, liver failure and pneumonia) and mortality within 30 days. As shown in Table 2, the results indicated that there was no significant difference in morbidity (including wound infection, bile leakage, liver failure and pneumonia) and mortality within 30 days (all $p>$ $0.05)$. Moreover, the mortality was stratified by DindoClavien classification (11), and the results also showed there were no significant differences between LLR and OLR groups $(p=0.990)$. In addition, compared to OLR, LLR obviously reduced postoperative hospital stay (6 vs. 9 days, $p=0.016$ ).

\subsection{Comparisons of the long-term OS and RFS}

All 43 patients received six courses of postoperative preventive chemotherapy, which consisted of Gemcitabine (Day 1, Day 8) plus S-1 (Day1-14), cycled 3 weeks 6 times. The median survival time, 1- and 3-year overall survival (OS) in LLR and OLR groups were 22.5 months, $76.9 \%$ and $47.1 \%$, and 12.1 months, $43.1 \%$ and $20.0 \%$, respectively (Figure 2A). The median survival, 1- and 3-year recurrence-free survival (RFS) in LLR and OLR groups were 10.3 months, $27.8 \%$ and $0 \%$, and 8.1 months, $24.0 \%$ and $4.0 \%$, respectively (Figure $2 \mathrm{~B}$ ).

The site of recurrence included liver, lymph node, incisional or abdominal implantation, bone, et al. There was no obvious difference in recurrent site and rate between LLR and OLR groups (Table 2). Multivariate

Table 1. Baseline characteristics of the included patients

\begin{tabular}{|c|c|c|c|}
\hline$N, \%$ or Median, IQR & LLR $(18,41.9 \%)$ & $\operatorname{OLR}(25,58.1 \%)$ & $p$ \\
\hline \multicolumn{4}{|l|}{ The patient- and liver-related variables } \\
\hline Age, years & $64(60-72)$ & $61(55-64)$ & 0.100 \\
\hline Sex, male & $12(67)$ & $10(40)$ & 0.084 \\
\hline Comorbid illnesses & $5(12)$ & $7(28)$ & 0.987 \\
\hline ASA score, $\leq 2$ & $15(83)$ & $19(76)$ & 0.839 \\
\hline Abdominal surgical history & $3(16)$ & $7(28)$ & 0.616 \\
\hline Total bilirubin, $>24 \mu \mathrm{mol} / \mathrm{L}$ & $3(16)$ & $8(32)$ & 0.648 \\
\hline \multicolumn{4}{|l|}{ The tumor-related variables } \\
\hline CA19-9 level, > $200 \mathrm{U} / \mathrm{mL}$ & $8(44)$ & $14(56)$ & 0.455 \\
\hline CEA level, $>5 \mu \mathrm{g} / \mathrm{L}$ & $7(39)$ & $10(40)$ & 0.914 \\
\hline \multicolumn{4}{|l|}{ TNM stage } \\
\hline $\mathrm{IA}+\mathrm{IB}$ & 7 (39) & $17(68)$ & 0.058 \\
\hline II +III & $11(61)$ & $8(32)$ & \\
\hline Nerve invasion & $4(22)$ & $9(36)$ & 0.332 \\
\hline Vascular invasion & $4(22)$ & $9(36)$ & 0.332 \\
\hline Tumor differentiation, poor & $14(78)$ & $17(68)$ & 0.480 \\
\hline \multicolumn{4}{|l|}{ The operative variables } \\
\hline Range of hepatectomy, major & $6(33)$ & $13(52)$ & 0.224 \\
\hline Number of lymphadenectomy, $\geq 6$ & $6(33)$ & $8(32)$ & 0.927 \\
\hline Intraoperative blood loss, $\mathrm{mL}$ & $375(275-500)$ & $500(350-750)$ & 0.016 \\
\hline Length of surgery, min & $305(207-390)$ & $300(257-392)$ & 0.730 \\
\hline
\end{tabular}


Table 2. Comparing the short and long-term outcomes between laparoscopic versus open liver resection

\begin{tabular}{|c|c|c|c|}
\hline$N, \%$ or Median, IQR & LLR $(18,41.9 \%)$ & $\operatorname{OLR}(25,58.1 \%)$ & $p$ \\
\hline Mortality within 30 days & $0(0)$ & $1(4)$ & 1.000 \\
\hline Postoperative hospital stays, days & $6(5-12)$ & $9(7-15)$ & 0.001 \\
\hline \multicolumn{4}{|l|}{ Postoperative Complication } \\
\hline Wound Infection & $0(0)$ & $2(8)$ & 0.502 \\
\hline Bile Leakage & $1(6)$ & $2(8)$ & 1.000 \\
\hline Liver Failure & $0(0)$ & $1(4)$ & 1.000 \\
\hline Pneumonia & $2(11)$ & $2(8)$ & 1.000 \\
\hline \multicolumn{4}{|l|}{ Dindo-Clavien classification } \\
\hline $1-2$ & $17(94)$ & $23(92)$ & 0.990 \\
\hline $3-4$ & $1(6)$ & $2(8)$ & 1.000 \\
\hline Median OS, months & 22.5 & 12.1 & 0.073 \\
\hline 1 -year OS, $\%$ & 76.9 & 43.1 & 0.177 \\
\hline 3-year OS, \% & 47.1 & 20.0 & 0.819 \\
\hline Median RFS, months & 10.3 & 8.1 & 0.409 \\
\hline 1 -year RFS, \% & 27.8 & 24.0 & 0.348 \\
\hline 3-year RFS, \% & 0 & 4.0 & 0.750 \\
\hline \multicolumn{4}{|l|}{ Postoperative Recurrence Site } \\
\hline Liver & $7(39)$ & $11(44)$ & 0.738 \\
\hline Lymph Node & $3(17)$ & $3(12)$ & 1.000 \\
\hline Incisional or abdominal Implantation & $4(22)$ & $3(12)$ & 1.000 \\
\hline Bone & $1(6)$ & $4(14)$ & 0.567 \\
\hline Others & $2(11)$ & $3(12)$ & 0.990 \\
\hline
\end{tabular}
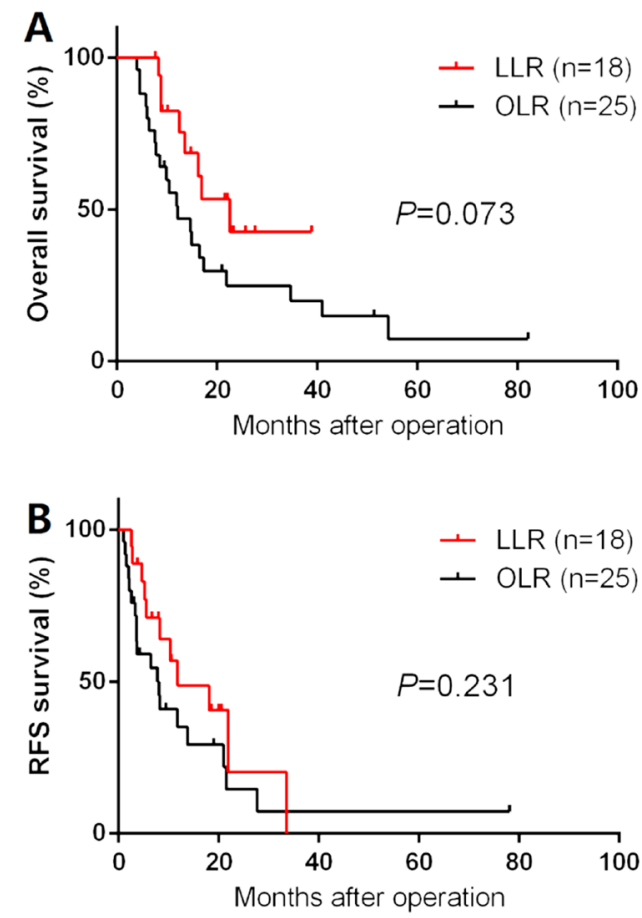

Figure 2. Comparisons of overall survival (A) and recurrence-free survival (B) curves between LLR and OLR groups.

analysis showed that CA19-9 level, TNM stage, and tumor differentiation were independent risk factors for the OS (Table 3) and RFS (Table 4).

3.4. Subgroup analysis of overall survival between LLR and OLR groups

We further analyzed the OS stratified by TNM stage
(IA+IB, II+III), CA19-9 ( $\geq 200 \mathrm{U} / \mathrm{mL},<200 \mathrm{U} /$ $\mathrm{mL})$, number of lymphadenectomy $(\geq 6,<6)$ and differentiation grade (well or moderately, poor). The cutoff points of CA19-9 and number of dissected lymph nodes were set at $200 \mathrm{U} / \mathrm{mL}$ and 6 respectively, which were considered as a high-risk factor and recommended number for postoperative staging (12) (Figure 3). The results from the subgroup analysis showed there were no significant differences between LLR and OLR groups (all $p>0.05)$.

\section{Discussion}

In the present study, 43 patients with ICC were retrospectively analyzed, who received liver resection and associated lymphadenectomy, and postoperative adjuvant chemotherapy in our hospital. The results showed that LLR obviously reduced intraoperative blood loss and postoperative hospital stay. Moreover, there was no significant difference in the short-term outcomes including postoperative morbidity (including wound infection, bile leakage, liver failure and pneumonia) and mortality within 30 days, and long-term outcomes (including OS and RFS) between LLR and OLR (all $p>$ 0.05). Multivariate analysis showed that CA19-9 level, TNM stage, and tumor differentiation were independent risk factors for the OS and RFS.

The technology of laparoscopy has evolved rapidly in recent years, ultra-high definition (UHD) camera and display system and electrosurgical instruments were employed in the surgery, which could provide a clear field and better hemostatic control for the surgeons. By this Amplifying effect of laparoscopy, LLR achieved less intraoperative blood loss than OLR. 
Table 3. Univariable and multivariable Cox-regression analyses on risk factors of overall survival

\begin{tabular}{|c|c|c|c|c|c|}
\hline \multirow{2}{*}{ Variables } & \multirow{2}{*}{ Comparison } & \multicolumn{2}{|l|}{ UV } & \multicolumn{2}{|l|}{ MV } \\
\hline & & HR $(95 \% \mathrm{CI})$ & $p$ & HR $(95 \% \mathrm{CI})$ & $p$ \\
\hline Age, years & continuous, years & $0.965(0.916-1.016)$ & 0.173 & & \\
\hline Sex, male & male $v s$. female & $1.403(0.672-2.928)$ & 0.367 & & \\
\hline Comorbid illnesses & with vs. without & $0.556(0.267-1.157)$ & 0.116 & & \\
\hline ASA score & $>2 v s . \leq 2$ & $0.822(0.332-2.036)$ & 0.671 & & \\
\hline Abdominal surgical history & with vs. without & $1.253(0.596-2.642)$ & 0.550 & & \\
\hline Total bilirubin & $>24 v s . \leq 24 \mathrm{mmol} / \mathrm{L}$ & $1.047(0.474-2.309)$ & 0.910 & & \\
\hline CA19-9 level & $>200 \mathrm{vs} . \leq 200 \mathrm{U} / \mathrm{mL}$ & $4.445(1.791-11.034)$ & 0.001 & $2.219(1.632-3.017)$ & $<0.001$ \\
\hline CEA level & $>5 v s . \leq 5 \mu \mathrm{g} / \mathrm{L}$ & $1.545(0.742-3.204)$ & 0.246 & & \\
\hline TNM stage & $\mathrm{II}+\mathrm{III} v s . \mathrm{IA}+\mathrm{IB}$ & $2.357(1.091-5.092)$ & 0.029 & $2.098(1.671-2.634)$ & $<0.001$ \\
\hline Nerve invasion & with $v s$. without & $1.590(0.740-3.416)$ & 0.235 & & \\
\hline Vascular invasion & with vs. without & $2.264(0.990-5.180)$ & 0.053 & & \\
\hline Tumor differentiation & poor $v s$. well or moderately & $2.865(1.261-6.513)$ & 0.012 & $1.524(1.093-2.126)$ & 0.013 \\
\hline Range of hepatectomy & major vs. minor & $0.884(0.422-1.851)$ & 0.743 & & \\
\hline Number of lymphadenectomy & $<6 v s . \geq 6$ & $1.916(0.893-4.109)$ & 0.095 & NS & \\
\hline Intraoperative blood loss & continuous, $\mathrm{mL}$ & $0.999(0.996-1.012)$ & 0.532 & & \\
\hline Length of surgery & continuous, $\min$ & $1.000(0.998-1.0)$ & 0.905 & & \\
\hline
\end{tabular}

"Those variables found significant at $P<0.10$ in univariable analyses were entered into multivariable analyses. HR, hazard ratio; UV, univariable; $\mathrm{MV}$, multivariable; CI, Confidence interval; NS, no significance.

Table 4. Univariable and multivariable Cox-regression analyses on risk factors of recurrence-free survival

\begin{tabular}{|c|c|c|c|c|c|}
\hline \multirow{2}{*}{ Variables } & \multirow{2}{*}{ Comparison } & \multicolumn{2}{|l|}{ UV } & \multicolumn{2}{|l|}{ MV } \\
\hline & & $\mathrm{HR}(95 \% \mathrm{CI})$ & $p$ & $\mathrm{HR}(95 \% \mathrm{CI})$ & $p$ \\
\hline Age, years & continuous, years & $0.964(0.897-1.003)$ & 0.169 & & \\
\hline Sex, male & male $v s$. female & $1.401(0.603-2.549)$ & 0.369 & & \\
\hline Comorbid illnesses & with vs. without & $0.555(0.371-1.640)$ & 0.115 & & \\
\hline ASA score & $>2 v s . \leq 2$ & $0.821(0.370-2.123)$ & 0.670 & & \\
\hline Abdominal surgical history & with vs. without & $1.253(0.371-1.719)$ & 0.552 & & \\
\hline Total bilirubin & $>24$ vs. $\leq 24 \mathrm{mmol} / \mathrm{L}$ & $1.046(0.414-2.127)$ & 0.912 & & \\
\hline CA19-9 level & $>200 v s . \leq 200 \mathrm{U} / \mathrm{mL}$ & $4.438(1.261-6.830)$ & 0.001 & $3.405(2.684-4.318)$ & $<0.001$ \\
\hline CEA level & $>5 v s . \leq 5 \mu \mathrm{g} / \mathrm{L}$ & $1.545(0.784-3.476)$ & 0.244 & & \\
\hline TNM stage & $\mathrm{II}+\mathrm{III} v s . \mathrm{IA}+\mathrm{IB}$ & $2.362(0.964-4.212)$ & 0.029 & $2.268(1.840-2.795)$ & 0.001 \\
\hline Nerve invasion & with $v s$. without & $1.587(0.749-3.171)$ & 0.236 & & \\
\hline Vascular invasion & with vs. without & $2.260(0.741-3.640)$ & 0.053 & & \\
\hline Tumor differentiation & poor $v s$. well or moderately & $2.861(1.172-5.715)$ & 0.012 & $1.885(1.485-2.392)$ & 0.029 \\
\hline Range of hepatectomy & major vs. minor & $0.885(0.498-2.114)$ & 0.747 & & \\
\hline Number of lymphadenectomy & $<6 v s . \geq 6$ & $1.913(1.303-6.236)$ & 0.096 & NS & \\
\hline Intraoperative blood loss & continuous, $\mathrm{mL}$ & $0.999(0.996-1.010)$ & 0.528 & & \\
\hline Length of surgery & continuous, min & $1.000(0.998-1.002)$ & 0.898 & & \\
\hline
\end{tabular}

"Those variables found significant at $P<0.1$ in univariable analyses were entered into multivariable analyses. HR, hazard ratio; UV, univariable; MV, multivariable; CI, Confidence interval; NS, no significance.

Masateru et al. reported intraoperative blood loss of LLR was less than OLR in patients of hepatocellular carcinoma with liver cirrhosis (13). Likewise, a similar outcome was confirmed by Cai et al., who compared 145 cases of LLR with 190 cases OLR in recurrent hepatocellular carcinoma (14). Hadrien et al. suggested a pneumoperitoneum of $10-14 \mathrm{mmHg}$ should be used as it allows good control of the bleeding, by which positive abdominal pressure could be seen as a factor to reduce intraoperative blood loss in LLR (15). More and more authors have reported their experience on LLR for ICC, and most of them have achieved satisfactory results, or at least not inferior $(9,16)$. Billy et al. reported 6 patients with stage I and 5 patients with stage II/III $\left(7^{\text {th }}\right.$
AJCC), who underwent laparoscopic liver resection and selectively LND. Finally, it suggested non-inferior oncological outcomes compared with 26 cases LLR (17 cases of stage I, 9 cases of stage II/III) (8) . In our study, 11 patients in stage I and 17 patients in stage I were involved in LLR and OLR groups respectively. and 7 patients underwent LLR and 8 patients underwent OLR in stage II/III. Moreover, all patients of LLR and OLR received regional lymphadenectomy (removed hepatoduodenal ligament lymph nodes). As a result, OS of LLR with regular lymphadenectomy group achieved identical outcomes with OLR compared with regular lymphadenectomy group, not only in the patients in stage I, but also those in stage II/III. 

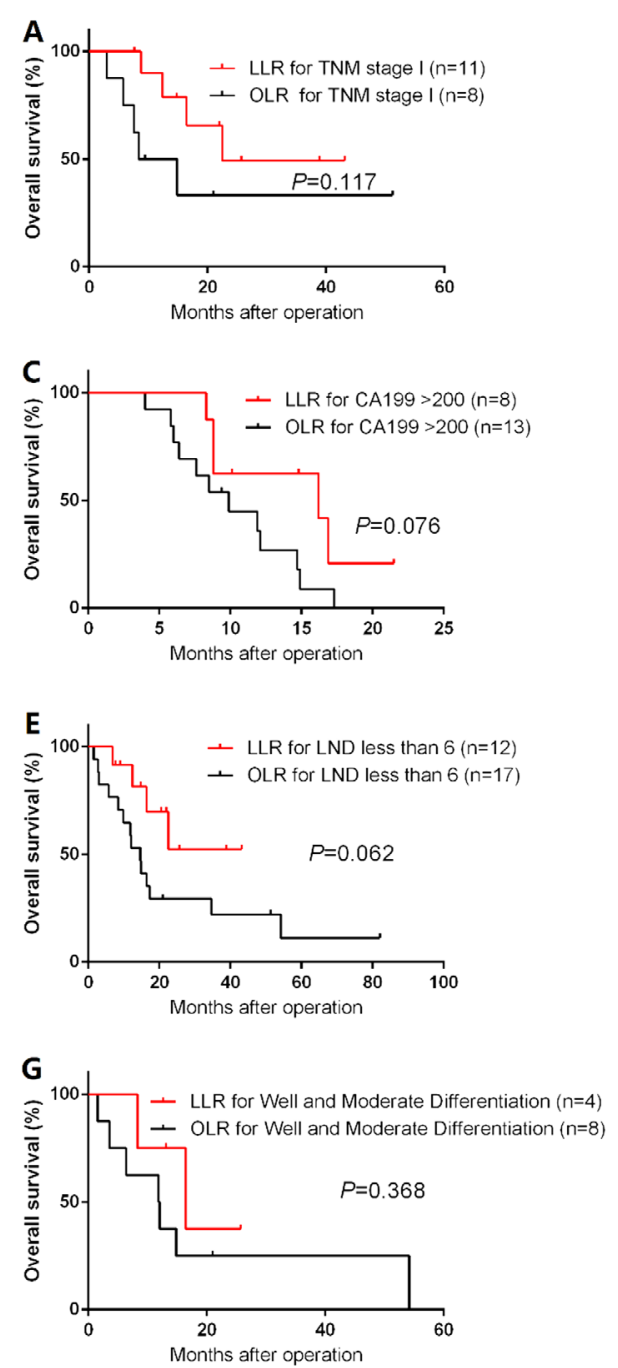
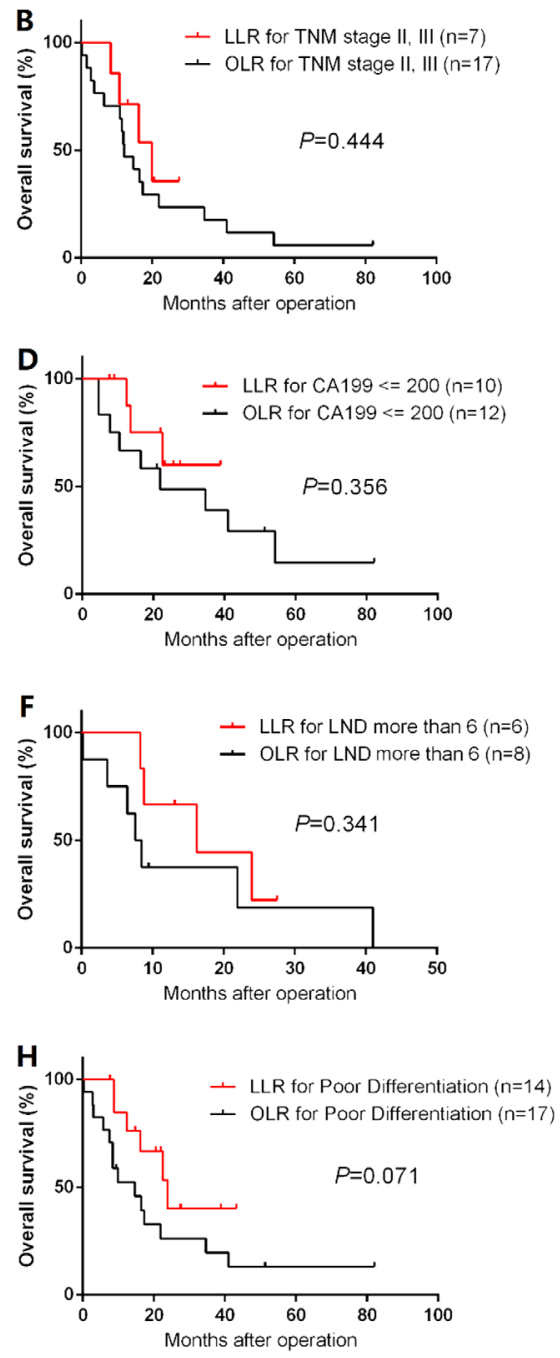

Figure 3. Subgroup analysis of overall survival stratified by TNM stage, CA19-9, number of lymphadenectomy and tumor differentiation.

Some published papers have demonstrated TNM stage, tumor differentiation, preoperative and CA199 level as important determinants of prognosis $(9,17)$. The present study also indicated that TNM stage, tumor differentiation and preoperative CA19-9 level were independent risk factors associated with poor survival. We further analyzed the difference stratified by TNM stage, tumor differentiation and preoperative CA199 level between LLR and OLR groups. The results indicated that these risk factors did not influence the prognosis between LLR and OLR groups.

Whether to perform lymphadenectomy and the range of lymphadenectomy in LLR are still controversial. Li et al. suggested ICC patients without lymph node involvement and patients with multiple tumors and lymph node metastases may not benefit from aggressive lymphadenectomy (18). Consensus statement from AHPBA declared that regional lymphadenectomy should be considered a standard part of surgical therapy for patients undergoing resection of ICC (19), because the incidence of nodal disease was high, with some studies showing lymph node metastasis in as many as $40 \%$ of patients $(17,20,21)$. However, some studies suggested that lymphadenectomy did not significantly improve prognosis, however, lymphadenectomy might be useful for nodal staging $(22,23)$. Based on this experience, we performed lymphadenectomy regularly, and the results in this study also demonstrated that increasing retrieved lymph node count or extended lymph node dissection was not associated with a survival benefit among patients who underwent curative surgical resection for ICC. Lymph node status, however, was prognostically important as patients with lymph node metastasis had a markedly worse long-term prognosis. Retrieval of 6 lymph nodes at the time of surgery was associated with the identification of more lymph node positive patients and therefore should be used as the goal cut-off value to avoid under-staging patients with ICC. In the aspect of range of lymphadenectomy, consensus of AHPBA recommended that lymph nodes of hepatoduodenal ligament (site 12) and common hepatic duct (site 8a) should be removed in 2015 . 
Besides, for ICC originating from right hemiliver, the retropancreatic lymph nodes (site 13) may be involved; if ICC is located in left hemiliver, in addition to abovementioned lymph nodes, the nodes around the cardiac portion of the stomach and along the lesser curvature (site 1 and 3) should also be removed. Similarly, Chinese experts recommended that lymphadenectomy for ICC should cover site 8, 12 and 13, which was suggested by Chinese Society of Clinical Oncology (CSCO) in 2019. The deadline for the cases we studied was 2017 , and the majority of our cases (31/43) underwent their surgery before those consensuses were issued. To the best of our knowledge at that time, range of LND was limited in site 12 .

CA19-9 $\geq 200 \mathrm{U} / \mathrm{mL}$ was identified as a negative prognostic factor in patients without preoperative jaundice, according to $8^{\text {th }}$ AJCC guideline. In our study, 18 patients in LLR group and 25 patients in OLR group divided into two subgroups by setting the cut-off point of CA19-9 at 200U/mL. OS in LLR group did not differ with the OLR group with and without this high-risk feature. Elevated preoperative CA19-9, thus, should not be considered as a contraindication of LLR for ICC patients. Poor differentiation has been demonstrated as another independent risk factor for prognosis. The underling mechanism may be that this kind of tumor cells are more likely to metastasize (24).

This study had several limitations. First, this study is not a randomized controlled trail and therefore biases in patient's selection may exist. Second, to make the results robust, only 43 patients were included. However, all patients were carefully selected to include only those with associated lymphadenectomy and postoperative adjuvant chemotherapy. Third, though all the patients received associated lymphadenectomy, we did not further analyze the number and range of positive lymph nodes. Fourth, though all the patients received 6 course of adjuvant chemotherapy, we did not further analyze the role and complication of chemotherapy, especially for different TNM stage. Fifth, there was no statistically significant difference in OS or RFS between the two groups in this study. However, the sample size of both groups was small, and the follow-up time of LLR group was short. Sixth, LLR has achieved equal OS with OLR, but proportion of TNM stage II/III of LLR was higher than OLR $(61.1 \% v s .32 .0 \%, p>0.05)$. Statistically, there was no significant difference between the two groups, this phenomenon may be associated with small-size sample, which could be affected by extremum easily. Moreover, shorter follow-up time of LLR was likely the cause.

In conclusion, the present study demonstrated LLR for ICC is safe and feasible compared with OLR. The advantage for LLR was to reduce intraoperative blood loss and postoperative hospital stay. Furthermore, future randomized controlled trials are still needed to better define the role of LLR.

\section{References}

1. Bergquist A, von Seth E. Epidemiology of cholangiocarcinoma. Best Pract Res Clin Gastroenterol. 2015; 29:221-232.

2. Bridgewater J, Galle PR, Khan SA, Llovet JM, Park JW, Patel T, Pawlik TM, Gores GJ. Guidelines for the diagnosis and management of intrahepatic cholangiocarcinoma. J Hepatol. 2014; 60:1268-1289.

3. Wang Y, Li J, Xia Y, Gong R, Wang K, Yan Z, Wan X, Liu G, Wu D, Shi L, Lau W, Wu M, Shen F. Prognostic nomogram for intrahepatic cholangiocarcinoma after partial hepatectomy. J Clin Oncol. 2013; 31:1188-1195.

4. Hwang S, Lee YJ, Song GW, Park KM, Kim KH, Ahn CS, Moon DB, Lee SG. Prognostic Impact of Tumor Growth Type on $7^{\text {th }}$ AJCC Staging System for Intrahepatic Cholangiocarcinoma: a Single-Center Experience of 659 Cases. J Gastrointest Surg. 2015; 19:1291-1304.

5. Hashizume M, Takenaka K, Yanaga K, Ohta M, Kajiyama K, Shirabe K, Itasaka H, Nishizaki T, Sugimachi K. Laparoscopic hepatic resection for hepatocellular carcinoma. Surg Endosc. 1995; 9:1289-1291.

6. Kaneko H, Takagi S, Shiba T. Laparoscopic partial hepatectomy and left lateral segmentectomy: technique and results of a clinical series. Surgery. 1996; 120:468475.

7. Ciria R, Cherqui D, Geller DA, Briceno J, Wakabayashi G. Comparative Short-term Benefits of Laparoscopic Liver Resection: 9000 Cases and Climbing. Ann Surg. 2016; 263:761-777.

8. Uy BJ, Han HS, Yoon YS, Cho JY. Laparoscopic liver resection for intrahepatic cholangiocarcinoma. J Laparoendosc Adv Surg Tech A. 2015; 25:272-277.

9. Lee W, Park JH, Kim JY, Kwag SJ, Park T, Jeong SH, Ju YT, Jung EJ, Lee YJ, Hong SC, Choi SK, Jeong CY. Comparison of perioperative and oncologic outcomes between open and laparoscopic liver resection for intrahepatic cholangiocarcinoma. Surg Endosc. 2016; 30:4835-4840.

10. Kinoshita M, Kanazawa A, Takemura S, Tanaka S, Kodai S, Shinkawa H, Shimizu S, Murata A, Nishio K, Hamano G, Ito T, Tsukamoto T, Kubo S. Indications for laparoscopic liver resection of mass-forming intrahepatic cholangiocarcinoma. Asian J Endosc Surg. 2020; 13:46-58.

11. Dindo D, Clavien PA. What is a surgical complication? World J Surg. 2008; 32:939-941.

12. Lee AJ, Chun YS. Intrahepatic cholangiocarcinoma: the AJCC/UICC $8^{\text {th }}$ edition updates. Chin Clin Oncol. 2018; 7:52.

13. Yamamoto M, Kobayashi T, Oshita A, Abe T, Kohashi T, Onoe T, Fukuda S, Omori I, Imaoka Y, Honmyo N, Ohdan H. Laparoscopic versus open limited liver resection for hepatocellular carcinoma with liver cirrhosis: a propensity score matching study with the Hiroshima Surgical study group of Clinical Oncology (HiSCO). Surg Endosc. 2019.

14. Cai W, Liu Z, Xiao Y, Zhang W, Tang D, Cheng B, Li Q. Comparison of clinical outcomes of laparoscopic versus open surgery for recurrent hepatocellular carcinoma: a meta-analysis. Surg Endosc. 2019; 33:3550-3557.

15. Tranchart H, O'Rourke N, Van Dam R, Gaillard M, Lainas P, Sugioka A, Wakabayashi G, Dagher I. Bleeding control during laparoscopic liver resection: a review of literature. J Hepatobiliary Pancreat Sci. 2015; 22:371-378.

16. Waisberg DR, Pinheiro RS, Nacif LS, Rocha-Santos V, Martino RB, Arantes RM, Ducatti L, Lai Q, Andraus 
W, D'Albuquerque LC. Resection for intrahepatic cholangiocellular cancer: new advances. Transl Gastroenterol Hepatol. 2018; 3:60.

17. Ribero D, Pinna AD, Guglielmi A, et al. Surgical Approach for Long-term Survival of Patients With Intrahepatic Cholangiocarcinoma: A Multi-institutional Analysis of 434 Patients. Arch Surg. 2012; 147:11071113.

18. Li DY, Zhang HB, Yang N, Quan Y, Yang GS. Routine lymph node dissection may be not suitable for all intrahepatic cholangiocarcinoma patients: results of a monocentric series. World J Gastroenterol. 2013; 19:90849091.

19. Weber SM, Ribero D, O'Reilly EM, Kokudo N, Miyazaki M, Pawlik TM. Intrahepatic cholangiocarcinoma: expert consensus statement. HPB (Oxford). 2015; 17:669-680.

20. Luo X, Yuan L, Wang Y, Ge R, Sun Y, Wei G. Survival outcomes and prognostic factors of surgical therapy for all potentially resectable intrahepatic cholangiocarcinoma: a large single-center cohort study. J Gastrointest Surg. 2014; 18:562-572.

21. Endo I, Gonen M, Yopp AC, et al. Intrahepatic cholangiocarcinoma: rising frequency, improved survival, and determinants of outcome after resection. Ann Surg. 2008; 248:84-96.

22. Shimada M, Yamashita Y, Aishima S, Shirabe K,
Takenaka K, Sugimachi K. Value of lymph node dissection during resection of intrahepatic cholangiocarcinoma. $\mathrm{Br} \mathrm{J}$ Surg. 2001; 88:1463-1466.

23. Kim DH, Choi DW, Choi SH, Heo JS, Kow AW. Is there a role for systematic hepatic pedicle lymphadenectomy in intrahepatic cholangiocarcinoma? A review of 17 years of experience in a tertiary institution. Surgery. 2015; 157:666-675.

24. Blok P, Craanen ME, Dekker W, Tytgat GN. Loss of E-cadherin expression in early gastric cancer. Histopathology. 1999; 34:410-415.

Received August 7, 2020; Revised September 4, 2020; Accepted September 8, 2020.

${ }^{\S}$ These authors contributed equally to this work.

*Address correspondence to:

Chengwu Zhang, Department of Hepatobiliary and Pancreatic Surgery \& Minimally Invasive Surgery, Zhejiang Provincial People's Hospital \& Hangzhou Medical College affiliated People's Hospital, 158 Shangtang RD, Hangzhou 310014, Zhejiang, China.

E-mail: zcwzry@163.com

Released online in J-STAGE as advance publication September 11, 2020. 